Technical Note

\title{
On the exponential stability of a flexible structure in thermo-elasticity with micro-temperature effects
}

\author{
Mohamed HOUASNI ${ }^{1, *}$ (i), Salah ZITOUNI ${ }^{2}$,, Abdelhak DJEBABLA ${ }^{3}(1)$ \\ ${ }^{1}$ Faculté des Sciences et de la Technologie, Université DBKM, Algérie \\ ${ }^{2}$ Department of Mathematics and Informatics, Souk AhrasUniv, Souk Ahras, Algeria \\ ${ }^{3}$ Laboratory of Applied Mathematics, University BadjiMokhtar, Annaba, Algeria
}

\section{ARTICLE INFO}

Article history

Received: 16 August 2020

Accepted: 14 December 2020

\section{Key words:}

Decay; Flexible structure;

Semigroups theory; Exponential

stability; Micro-temperature

damping

\begin{abstract}
In this paper, we consider a non-uniform flexible structure with micro-temperature effect. We prove the well-posed of the problem using semi-group theory, as well as an exponential stability using the multiplier method without any restriction or relation on the coefficients of the system.
\end{abstract}

Cite this article as: Mohamed $\mathrm{H}$, Salah Z, Abdelhak D. On the exponential stability of a flexible structure in Thermo-Elasticity with Micro-Temperature effects. Sigma J Eng Nat Sci 2021;39(3):260-267.

\section{INTRODUCTION}

In this paper, we aim to study the following inhomogeneous flexible structure system with micro-temperature effects:

$$
\left\{\begin{array}{c}
m(x) u_{t t}-\left(p(x) u_{x}+2 \delta(x) u_{x t}\right)_{x}+d w_{x}+\eta \theta_{x}=0 \\
c \theta_{t}-k \theta_{x x}+\eta u_{x t}+k_{1} w_{x}=0 \\
\tau w_{t}-k_{3} w_{x x}+k_{2} w+k_{1} \theta_{x}+d u_{x t}=0
\end{array}\right.
$$

where $u(x, t)$ is the displacement of a particle at position $x \in(0, L)$ and time $t>0, \theta$ and $w$ are the temperature of the body and the micro-temperature vector respectively. $\eta>0$

\section{${ }^{*}$ Corresponding author.}

*E-mail address: n.houasni@gmail.com

This paper was recommended for publication in revised form by Regional Editor Mustafa Düldül is the coupling constant, that accounts for the heating effect, and $k, k_{1}, k_{2}, k_{3}, c, d, \tau>0 . m(x), \delta(x)$ and $p(x)$ are responsible for the non-uniform structure of the body, and, respectively, denote mass per unit length of structure, coefficient of internal material damping and a positive function related to the stress acting on the body at a point $x$. We consider the following initial and boundary conditions:

$$
\begin{aligned}
u(., 0) & =u_{0}(x), u_{t}(., 0)=u_{1}(x), \theta(., 0) \\
& =\theta(x), w(., 0)=w_{0}(x), \\
u(0, t) & =u(L, t)=\theta(0, t)=\theta(L, t)=w_{x}(0, t) \\
& =w_{x}(L, t)=0, \forall t \geq 0
\end{aligned}
$$


The issue of existence and stability of flexible structure system has attracted a great deal of attention in the last years. Misra et al. [20] considered the vibrations of a cantilever structure modeled by the standard linear flexible model of visco-elasticity coupled to an expectedly dissipative effect through heat conduction

$$
\left\{\begin{array}{c}
m(x) u_{t t}-\left(p(x) u_{x}+2 \delta(x) u_{x t}\right)_{x}-k \theta_{x}=f \\
\theta_{t}-\theta_{x x}-k u_{x t}=0
\end{array}\right.
$$

By using semigroups theory and multiplier technique, they established the well-posedness and an exponential stability of the system when the disturbing force is insignificant. In the presence of second sound, Alves et al. [2] concerned with the system;

$$
\left\{\begin{array}{c}
m(x) u_{t t}-\left(p(x) u_{x}+2 \delta(x) u_{x t}\right)_{x}+\eta \theta_{x}=0 \\
\theta_{t}+k q_{x}+\eta u_{x t}=0 \\
\tau q_{t}+\beta q+k \theta_{x}=0
\end{array}\right.
$$

They established the well-posedness of the system and proved its stability exponential and polynomial under suitable boundary conditions. Li et al. [18] considered this last with a delay term of the form $\mu u_{t}\left(x, t-\tau_{0}\right)$ in its first equation, they proved that the system is exponential decay under a "small" condition on time delay. For more details discussion on the subject see $[1,10]$ and the references therein.

Historically, the linear theory of thermo-elasticity with micro-temperatures for materials with inner structure whose particles, in addition to the classical displacement and temperature fields, possess micro-temperatures was constructed by Iesan and Quintanilla $[15,17]$. The work is motivated by increasing use of materials which possess thermal variation at a microstructure level. The same authors proved an existence theorem and established the continuous dependence of solutions of the initial data and body loads. We note that the concept of micro-temperature was just used in the theory of thermodynamics for elastic materials with microstructure. In addition to micro-deformations of the string, the micro-elements of the continuum possess micro-temperatures which represent the variation of the temperature within a micro-volume. Originally, Grot [11] was the first to take into consideration the inner structure of a body in order to develop a thermodynamic theory for thermo-elastic materials where micro-elements, in addition to classic micro-deformations, possess micro-temperatures. While, the fundamental solution of the equations of the theory of thermo-elasticity with micro-temperatures was constructed by Svanadze [27]. Riha [23, 24] developed a further study concerning heat conduction in thermo-elastic materials with inner structure. It is shown that the experimental data for the silicone rubber containing spherical aluminum particles and for human blood are conform closely to the predicted theoretical model of thermo-elasticity with micro-temperatures. We refer the interested readers to $[3,5,6,7,8,9,12,13,14,16,19,25,26]$ for details discussion on the theory.

Motivated by works mentioned above, we investigate (1)-(2) under suitable condition and establish the well-posedness of the problem using semi-group theory, as well as the stability result of the solution using the multiplier method. We should mention here that, to the best of our knowledge, there is no result concerning flexible structure system with micro-temperature effect. Our purpose in the present manuscript is to obtain an exponential decay rate estimates of the energy function of (1) without any restriction or relation on the coefficients of the system.

This paper is organized as follows; In the second section, we introduce some assumptions needed in our work then prove the well-posedness of the system (1) - (2). In the last section we state and prove our stability result.

\section{WELL-POSEDNESS OF THE PROBLEM}

In this section, we present some assumptions and give the existence and uniqueness result of system (1) - (2) using the semigroup theory. Throughout this paper, $c^{\prime}$ represents ageneric positive constant and is different in various occurrences.

The aim of this section is to prove that system (1) - (2) is well-posed. From Equation $(1)_{3}$ and the boundary conditions (2), we have

$$
\frac{d}{d t} \int_{0}^{L} w(x, t) d x+\frac{k_{2}}{\tau} \int_{0}^{L} w(x, t) d x=0, \forall t \geq 0,
$$

Thus

$$
\int_{0}^{L} w(x, t) d x=\left(\int_{0}^{L} w_{0} d x\right) \exp \left(\frac{-t}{\tau} k_{2}\right), \forall t \geq 0
$$

So, if we set

$$
\tilde{w}(x, t)=w(x, t)-\frac{1}{L}\left(\int_{0}^{L} w_{0} d x\right) \exp \left(\frac{-t}{\tau} k_{2}\right), \forall t \geq 0, x \in[0, L] .
$$

Then, $\left(u, u_{t}, \theta, \tilde{w}\right)$ satisfies Equations (1), and

$$
\int_{0}^{L} \tilde{w}(x, t) d x=0
$$


for all $t \geq 0$. In the sequel, we shall work with $\tilde{w}$ but we write $w$ for simplicity. The energy functional associated to (1) (2), namely,

$$
E\left(t, u, u_{t}, \theta, w\right)=\frac{1}{2} \int_{0}^{L}\left\{p(x) u_{x}^{2}+m(x) u_{t}^{2}+c \theta^{2}+\tau w^{2}\right\} d x
$$

we denote $\mathrm{E}(t)=E\left(t, u, u_{t}, \theta, w\right)$ and $E(0)=E\left(0, u_{0}\right.$, $u_{1}, \theta_{0}, w_{0}$ ) for simplicity of notations. Then the energy $E$ is decreasing function and satisfies, for all $t \geq 0$.

$$
\begin{aligned}
E^{\prime}(t)= & -2 \int_{0}^{L} \delta(x) u_{x t}^{2} d x-k_{2} \int_{0}^{L} w^{2} d x-k_{3} \int_{0}^{L} w_{x}^{2} d x-k \int_{0}^{L} \theta_{x}^{2} d x \\
& \leq-c^{\prime} \int_{0}^{L} u_{t}^{2} d x-k_{2} \int_{0}^{L} w^{2} d x-k_{3} \int_{0}^{L} w_{x}^{2} d x-k \int_{0}^{L} \theta_{x}^{2} d x \leq 0 .
\end{aligned}
$$

To obtain precise decay rates of $E(t)$ as $t \rightarrow+\infty$, we assume that

$\mathrm{m}, \delta, p \in W^{1, \infty}(0, L), m(x), p(x)$ and $\delta(x)>0, \forall x \in[0, L]$.

Let us introducing the vector function $U=(u, v, \theta, w)^{\mathrm{T}}$, where $v=u_{t}$, using the standard Lebesgue space $L^{2}(0, L)$ and the Sobolev space $H_{0}^{1}(0, L)$ with their usual scalar products and norms for define the spaces;

$$
\check{H}=H_{0}^{1}(0, L) \times\left[\mathrm{L}^{2}(0, L)\right]^{2} \times L_{*}^{2}(0, L),
$$

And

$$
H_{*}^{2}(0, L)=\left\{w \in H^{2}(0, L): w_{x}(L)=w_{x}(0)=0\right\},
$$

Where

$$
L_{*}^{2}(0, L)=\left\{\omega \in L^{2}(0, L): \int_{0}^{L} \omega(s) d s=0\right\} .
$$

We equip $\check{\mathrm{H}}$ with the inner product:

$$
(U, \tilde{U})_{\breve{H}}=\int_{0}^{L} p(x) u_{x} \tilde{u}_{x} d x+\int_{0}^{L} m(x) v \tilde{v} d x+c \int_{0}^{L} \theta \tilde{\theta} d x+\tau \int_{0}^{L} w \tilde{w} d x .
$$

Next, the system (1) - (2) can be reduced to the following abstract Cauchy problem;

$$
\left\{\begin{array}{cl}
U^{\prime}(t)+A U(t)=0 & t>0 \\
U(0)=U_{0}=\left(u_{0}, u_{1}, \theta_{0}, w_{0}\right)^{T} &
\end{array}\right.
$$

where the operator $A: D(A) \rightarrow$ H is defined by

$$
A U=\left(\begin{array}{c}
-v \\
-\frac{1}{m(x)}\left(p(x) u_{x}+2 \delta(x) v_{x}-d w-\eta \theta\right)_{x} \\
\frac{1}{c}\left(-k \theta_{x x}+\eta u_{x t}+k_{1} w_{x}\right) \\
\frac{1}{\tau}\left(-k_{3} w_{x x}+k_{2} w+k_{1} \theta_{x}+d u_{x t}\right)
\end{array}\right) .
$$

The domain of $A$ is then

$$
A U=\left\{\begin{array}{c}
U \in \breve{H}: u \in H^{2}(0, L) \cap H_{0}^{1}(0, L), \\
v \in H_{0}^{1}(0, L), \theta \in H^{2}(0, L) \\
w \in L_{*}^{2}(0, L) \cap H_{*}^{2}(0, L)
\end{array}\right\},
$$

which is dense in $\check{H}$.

Proposition 2.1. Let $U_{0} \in \check{H}$ be given. Problem (6) possesses then a unique solution satisfying $U \in C\left(\mathrm{R}^{+}, \check{H}\right)$. If $U_{0} \in D(A)$ then $U \in C^{1}\left(R^{+}, \check{H}\right) \cap C\left(R^{+}, D(A)\right)$.

Proof. For any $U \in D(A)$, we have

$$
\begin{aligned}
(A U, U)_{\breve{H}}= & 2 \int_{0}^{L} \delta(x) v_{x}^{2} d x+k \int_{0}^{L} \theta_{x}^{2} d x \\
& +k_{2} \int_{0}^{L} w^{2} d x+k_{3} \int_{0}^{L} w_{x}^{2} d x \geq 0 .
\end{aligned}
$$

Hence, $A$ is monotone. Next, we prove that the operator $I+A$ is surjective.

Given $G=\left(g_{1}, g_{2}, g_{3}, g_{4}\right)^{\mathrm{T}} \in \breve{H}$, we prove that there exists $U \in D(A)$ satisfying

$$
(I+A) U=G
$$

which gives

$$
\begin{gathered}
-v+u=g_{1} \in H_{0}^{1}(0, L), \\
-\left(p(x) u_{x}+2 \delta(x) v_{x}-d w-\eta \theta\right)_{x}+m(x) v \\
=m(x) g_{2} \in L^{2}(0, L), \\
-k \theta_{x x}+\eta v_{x}+k_{1} w_{x}+c \theta=c g_{3} \in L^{2}(0, L), \\
-k_{3} w_{x x}+k_{2} w+k_{1} \theta_{x}+d v_{x}+\tau w=\tau g_{4} \in L_{\star}^{2}(0, L) .
\end{gathered}
$$

Inserting $v=u-g_{1}$, in $(8)_{2},(8)_{3}$ and $(8)_{4}$ we obtain

$$
\begin{gathered}
-\left(p(x) u_{x}+2 \delta(x) u_{x}-d w-\eta \theta\right)_{x}+m(x) u \\
=m(x)\left(g_{1}+g_{2}\right)-2 \delta(x) g_{1 x x}=f_{1} \in L^{2}(0, L), \\
-k \theta_{x x}+\eta u_{x}+k_{1} w_{x}+c \theta=c g_{3}+\eta g_{1 x} \\
=f_{2} \in L^{2}(0, L), \\
-k_{3} w_{x x}+k_{2} w+k_{1} \theta_{x}+d u_{x}+\tau w=\tau g_{4}+d g_{1 x} \\
=f_{3} \in L_{*}^{2}(0, L) .
\end{gathered}
$$

The variational formulation corresponding to Equation (9) takes the form

$$
B((u, \theta, w),(\tilde{u}, \tilde{\theta}, \tilde{w}))=F((\tilde{u}, \tilde{\theta}, \tilde{w}))
$$


where $B:\left[H_{0}^{1}(0, L) \times L^{2}(0, L) \times L_{*}^{2}(0, L)\right]^{2} \rightarrow \mathbb{R}$ is the bilinear form defined by

$$
\begin{aligned}
& B((u, \theta, w),(\tilde{u}, \tilde{\theta}, \tilde{w}))= \\
& \int_{0}^{L}\left[(p(x)+2 \delta(x)) u_{x}-d w-\eta \theta\right] \tilde{u}_{x} d x \\
& +\int_{0}^{L} m(x) u \tilde{u} d x+k \int_{0}^{L} \theta_{x} \tilde{\theta}_{x} d x \\
& -\eta \int_{0}^{L} u \tilde{\theta}_{x} d x-k_{1} \int_{0}^{L} w \tilde{\theta}_{x} d x+k_{3} \int_{0}^{L} w_{x} \tilde{w}_{x} d x \\
& +\left(k_{2}+\tau\right) \int_{0}^{L} w \tilde{w} d x+k_{1} \int_{0}^{L} \theta_{x} \tilde{w} d x \\
& -d \int_{0}^{L} u \tilde{w}_{x} d x,
\end{aligned}
$$

and $F: H_{0}^{1}(0, L) \times L^{2}(0, L) \times L_{*}^{2}(0, L) \rightarrow \mathbb{R}$ is the linear form defined by

$$
F((\tilde{u}, \tilde{\theta}, \tilde{w}))=\int_{0}^{L} f_{1} \tilde{u} d x+\int_{0}^{L} f_{2} \tilde{\theta} d x+\int_{0}^{L} f_{3} \tilde{w} d x .
$$

For $V=H_{0}^{1}(0, L) \times L^{2}(0, L) \times L_{\star}^{2}(0, L)$ equipped with the norm

$$
\|(u, \theta, w)\|_{V}^{2}=\|u\|_{2}^{2}+\left\|u_{x}\right\|_{2}^{2}+\|w\|_{2}^{2}+\theta_{x} \|_{2}^{2}
$$

where $\|\cdot\|_{2}$ is the usual norm.

One can easily see that $B$ and $F$ are bounded. Also, we get

$$
\begin{aligned}
B((u, \theta, w),(u, \theta, w))= & \int_{0}^{L}(p(x)+2 \delta(x)) u_{x}^{2} d x \\
& +\int_{0}^{L} m(x) u^{2} d x+k \int_{0}^{L} \theta_{x}^{2} d x \\
& +k_{3} \int_{0}^{L} w_{x}^{2} d x+k_{2} \int_{0}^{L} w^{2} d x \\
\geq & c(u, \theta, w)_{V}^{2} .
\end{aligned}
$$

Then, $B$ is coercive. Consequently, by the Lax-Milgram lemma (see [4] Corollary 5.8), system (9) has a unique solution

$$
u \in H_{0}^{1}(0, L), \theta \in L^{2}(0, L), w \in L_{\star}^{2}(0, L) .
$$

From $(8)_{1}$, we infer that

$$
v \in \mathrm{H}_{0}^{1}(0, L),
$$

Moreover, if $(\tilde{\theta}, \tilde{w}) \equiv(0,0) \in L^{2}(0, L) \times L_{*}^{2}(0, L)$ then Equation (10) reduces to

$$
\begin{aligned}
& -\int_{0}^{L}\left[(p(x)+2 \delta(x)) u_{x}-d w-\eta \theta\right]_{x} \tilde{u} d x \\
& +\int_{0}^{L} m(x) u \tilde{u} d x=\int_{0}^{L} f_{1} \tilde{u} d x,
\end{aligned}
$$

That is

$-\left[(p(x)+2 \delta(x)) u_{x}\right]_{x}=d w_{x}+\eta \theta_{x}-m(x) u+f_{1} \in L^{2}(0, L)$.

Consequently, by the regularity theory for the linear elliptic equations, it follows that

$$
u \in H^{2}(0, L) \cap H_{0}^{1}(0, L) .
$$

Similary, if $(\tilde{u}, \tilde{\theta}) \equiv(0,0) \in H_{0}^{1}(0, L) \times L^{2}(0, L)$ then Equation (10) reduces to

$$
\begin{aligned}
& k_{3} \int_{0}^{L} w_{x} \tilde{w}_{x} d x+\left(k_{2}+\tau\right) \int_{0}^{L} w \tilde{w} d x+k_{1} \int_{0}^{L} \theta_{x} \tilde{w} d x-d \int_{0}^{L} u \tilde{w}_{x} d x \\
& =\int_{0}^{L} f_{3} \tilde{w} d x, \forall \tilde{w} \in L_{*}^{2}(0, L) .
\end{aligned}
$$

That is

$$
k_{3} w_{x x}=\left(k_{2}+\tau\right) w+k_{1} \theta_{x}+d u_{x}-f_{3} \in L^{2}(0, L) .
$$

then, it follows that $\int_{0}^{\mathrm{L}} w d x=0$, and we get

$$
\mathrm{w} \in L_{\star}^{2}(0, L) \cap H^{2}(0, L) .
$$

Moreover, (11) is also true for any $\varphi \in C^{1}([0, L]) \subset$ $L_{*}^{2}(0, L)$. Hence, we have

$$
\begin{aligned}
& k_{3} \int_{0}^{L} w_{x} \varphi_{x} d x+\left(k_{2}+\tau\right) \int_{0}^{L} w \varphi d x+k_{1} \int_{0}^{L} \theta_{x} \varphi d x-d \int_{0}^{L} u \varphi_{x} \\
& \quad=\int_{0}^{L} f_{3} \varphi d x,
\end{aligned}
$$

for all $\varphi \in C^{1}([0, L])$. Thus, using integration by parts and bearing in mind (12), we obtain

$$
w_{x}(L) \varphi(L)-w_{x}(0) \varphi(0)=0, \forall \varphi \in C^{1}([0, L]) .
$$

Therefore, $w_{x}(L)=w_{x}(0)=0$, consequently, we have

$$
\mathrm{w} \in L_{*}^{2}(0, L) \cap H_{*}^{2}(0, L) .
$$

Now, if $(\tilde{u}, \tilde{w}) \equiv(0,0) \in H_{0}^{1}(0, L) \times L_{*}^{2}(0, L)$, then Equation (10) reduces to

$$
k \int_{0}^{L} \theta_{x} \tilde{\theta}_{x} d x-\eta \int_{0}^{L} u \tilde{\theta}_{x} d x-k_{1} \int_{0}^{L} w \tilde{\theta}_{x} d x=\int_{0}^{L} f_{2} \tilde{\theta} d x
$$


That is

$$
-k \theta_{x x}=f_{2}-\eta u_{x}-k_{1} w_{x} \in L^{2}(0, L),
$$

then, we get

$$
\theta \in H^{2}(0, L)
$$

Hence, there exists a unique $U \in D(A)$ such that Equation (7) is satisfied. Consequently, $A$ is a maximal monotone operator. Then, $D(A)$ is dense in $\breve{H}$ (see Proposition 7.1 in [4]) and the result of Proposition 2.1 follows from LumerPhillips theorem (see [22]).

\section{EXPONENTIAL STABILITY}

In this section, we introduce some lemmas allow us to achieve our goal, which is the proof of the stability result.

Lemma 3.1. [21] (Poincaré type Scheeffer's inequality) Let $h \in H_{0}^{1}(0, L)$. Then it holds

$$
\int_{0}^{L}|h|^{2} d x \leq l \int_{0}^{L}\left|h_{x}\right|^{2} d x, l=\frac{L^{2}}{\pi^{2}}
$$

Lemma 3.2. $[2,20]$ Let $\left(u, u_{p} \theta, w\right)$ be the solution to system (1) - (2), with an initial datum in $D(A)$. Then, for any $t>0$, there exists a sequence of real numbers (depending on $t)$, denoted by $\xi_{i} \in[0, L],(i=1, \ldots, 6)$, such that:

$$
\begin{array}{ll}
\int_{0}^{L} p(x) u_{x}^{2} d x=p\left(\xi_{1}\right) \int_{0}^{L} u_{x}^{2} d x, & \int_{0}^{L} m(x) u_{t}^{2} d x=m\left(\xi_{2}\right) \int_{0}^{L} u_{t}^{2} d x, \\
\int_{0}^{L} m(x) u^{2} d x=m\left(\xi_{3}\right) \int_{0}^{L} u^{2} d x, & \int_{0}^{L} \delta(x) u^{2} d x=\delta\left(\xi_{4}\right) \int_{0}^{L} u^{2} d x, \\
\int_{0}^{L} \delta(x) u_{x}^{2} d x=\delta\left(\xi_{5}\right) \int_{0}^{L} u_{x}^{2} d x, & \int_{0}^{L} \delta(x) u_{x t}^{2} d x=\delta\left(\xi_{6}\right) \int_{0}^{L} u_{x t}^{2} d x .
\end{array}
$$

Lemma 3.3. Let $\left(u, u_{t}, \theta, w\right)$ be the solution to system (1) - (2), then the energy $E$ is non-increasing function and satisfies, for all $t \geq 0$,

$$
\begin{aligned}
E^{\prime}(t) & =\int_{0}^{L} \delta(x) u_{x t}^{2} d x-k_{2} \int_{0}^{L} w^{2} d x-k_{3} \int_{0}^{L} w_{x}^{2} d x-k \int_{0}^{L} \theta_{x}^{2} d x \\
\leq & -c^{\prime} \int_{0}^{L} u_{t}^{2} d x-k_{2} \int_{0}^{L} w^{2} d x-k_{3} \int_{0}^{L} w_{x}^{2} d x-k \int_{0}^{L} \theta_{x}^{2} d x \leq 0,
\end{aligned}
$$

where $c^{\prime}=2 \delta\left(\xi_{6}\right) / l$.

Proof. Multiplying the equations in $(1)_{1},(1)_{2}$, and $(1)_{3}$ by $u_{t}, \theta$ and $w$, respectively, integrate over $(0, L)$ and using (13), we obtain (14).

Lemma 3.4. The functional

$$
I_{1}(t)=\int_{0}^{L}\left(\delta(x) u_{x}^{2}+m(x) u_{t} u\right) d x
$$

Satisfies

$$
\begin{array}{r}
I_{1}^{\prime}(t) \leq-\left(p\left(\xi_{1}\right)-(\eta+d) \grave{o}_{1}\right) \int_{0}^{L} u_{x}^{2} d x+m\left(\xi_{2}\right) \\
\int_{0}^{L} u_{t}^{2} d x+\frac{\eta}{4 \grave{o}_{1}} \int_{0}^{L} \theta^{2} d x+\frac{d}{4 \grave{o}_{1}} \int_{0}^{L} w^{2} d x,
\end{array}
$$

for any $\in_{1}>0$

Proof. Differentiating Equation (15) with respect to $t$ and using Equations (1), we get

$$
I_{1}^{\prime}(t)=-\int_{0}^{L} p(x) u_{x}^{2} d x+m(x) \int_{0}^{L} u_{t}^{2} d x-\eta \int_{0}^{L} \theta_{x} u d x-d \int_{0}^{L} u w_{x} d x
$$

Using Young's inequality (see [4] p. 92), we have for $\epsilon_{1}>0$

$$
\begin{aligned}
& -\eta \int_{0}^{L} \theta_{x} u d x=\eta \int_{0}^{L} \theta u_{x} d x \leq \eta \int_{1} \int_{0}^{L} u_{x}^{2} d x+\frac{\eta}{4 \int_{1}} \int_{0}^{L} \theta^{2} d x \\
& -d \int_{0}^{L} u w_{x} d x=d \int_{0}^{L} w u_{x} d x \leq d \int_{1}^{L} \int_{0}^{L} u_{x}^{2} d x+\frac{d}{4 \int_{1}} \int_{0}^{L} w^{2} d x,
\end{aligned}
$$

application of Lemma 3.2 and the last two inequality completes the proof.

Lemma 3.5. The functional

$$
I_{2}(t)=\tau c \int_{0}^{L} \theta\left(\int_{0}^{x} w(y) d y\right) d x
$$

satisfies

$$
\begin{aligned}
& I_{2}^{\prime}(t) \leq\left(-k_{1} c+3 c^{\prime} \varepsilon_{2}\right) \int_{0}^{L} \theta^{2} d x+\frac{1}{2 \varepsilon_{2}} \int_{0}^{L} u_{t}^{2} d x+\frac{1}{4 \varepsilon_{2}} \int_{0}^{L} \theta_{x}^{2} d x \\
& +\left(k_{1} \tau+2 \varepsilon_{2} c^{\prime}+\frac{c^{\prime}}{4 \varepsilon_{2}}\right) \int_{0}^{L} w^{2} d x+\frac{1}{4 \varepsilon_{2}} \int_{0}^{L} w_{x}^{2} d x,
\end{aligned}
$$

for any $\epsilon_{1}>0$.

Proof. Taking the derivative of (17) and using (1) and $(1)_{3}$ we find

$$
\begin{aligned}
I_{2}^{\prime}(t) & =\tau\left(k \int_{0}^{L} \theta_{x x}\left(\int_{0}^{x} w(y) d y\right) d x-\eta \int_{0}^{L} u_{t x}\left(\int_{0}^{x} w(y) d y\right) d x\right) \\
& -k_{1} \tau \int_{0}^{L} w_{x}\left(\int_{0}^{x} w(y) d y\right) d x \\
& +c\left(k_{3} \int_{0}^{L} \theta\left(\int_{0}^{x} w_{y y}(y) d y\right) d x-k_{2} \int_{0}^{L} \theta\left(\int_{0}^{x} w(y) d y\right) d x\right) \\
& +c\left(-k_{1} \int_{0}^{L} \theta\left(\int_{0}^{x} \theta_{y}(y) d y\right) d x-d \int_{0}^{L} \theta\left(\int_{0}^{x} u_{t y}(y) d y\right) d x\right)
\end{aligned}
$$


Integration by parts and the fact that $\int_{0}^{L} w d x=0$, give us

$I_{2}^{\prime}(t)=\tau\left(k \int_{0}^{L} \theta_{x} w d x+\eta \int_{0}^{L} u_{t} w d x+k_{1} \int_{0}^{L} w^{2} d x\right)$

$+c\left(k_{3} \int_{0}^{L} \theta w_{x} d x-k_{2} \int_{0}^{L} \theta\left(\int_{0}^{x} w(y) d y\right) d x-k_{1} \int_{0}^{L} \theta^{2} d x-d \int_{0}^{L} \theta u_{t} d x,\right)$

using Young's inequality, we get also

$$
\begin{aligned}
& -k \int_{0}^{L} \theta_{x} w d x \leq \frac{1}{4 \grave{O}_{2}} \int_{0}^{L} \theta_{x}^{2} d x+c^{\prime} \varepsilon_{2} \int_{0}^{L} w^{2} d x \\
& \eta \int_{0}^{L} u_{t} w d x \leq \frac{1}{4 \grave{O}_{2}} \int_{0}^{L} u_{t}^{2} d x+c^{\prime} \varepsilon_{2} \int_{0}^{L} w^{2} d x \\
& k_{3} \int_{0}^{L} \theta w_{x} d x \leq \frac{1}{4 \grave{O}_{2}} \int_{0}^{L} w_{x}^{2} d x+c^{\prime} \varepsilon_{2} \int_{0}^{L} \theta^{2} d x \\
& -k_{2} \int_{0}^{L} \theta\left(\int_{0}^{x} w(y) d y\right) d x \leq c^{\prime} \varepsilon_{2} \int_{0}^{L} \theta^{2} d x+\frac{c^{\prime}}{4 \dot{O}_{2}} \int_{0}^{L} w^{2} d x \\
& \quad-d \int_{0}^{L} \theta u_{t} d x \leq \frac{1}{4 \grave{O}_{2}} \int_{0}^{L} u_{t}^{2} d x+c^{\prime} \varepsilon_{2} \int_{0}^{L} \theta^{2} d x
\end{aligned}
$$

From (19) and the inequalities (20) we infer (18).

Next, we define a Lyapunov functional $\mathcal{L}$ and show that it is equivalent to the energy functional.

Lemma 3.6. For $N$ sufficiently large, the functional defined by

$$
\mathcal{L}(t)=N E(t)+I_{1}(t)+N_{1} I_{2}(t)
$$

where $N$ and $N_{1}$ are positive real numbers to be chosen appropriately later, satisfies

$$
c_{1}^{\prime} E(t) \leq \mathcal{L}(t) \leq c_{2}^{\prime} E(t),
$$

where $c_{1}^{\prime}$ and $c_{2}^{\prime}$ are positive constants.

Proof. Let

$$
\wp(t)=I_{1}(t)+N_{1} I_{2}(t)
$$

then, exploiting Young's inequality, (13) and (3) we obtain

$$
\begin{array}{r}
|\wp(t)| \leq N_{1} \tau c \int_{0}^{L}\left|\theta\left(\int_{0}^{x} w(y) d y\right)\right| d x+\int_{0}^{L}\left(\delta(x) u_{x}^{2}+m(x)\left|u_{t} u\right|\right) d x \\
\leq \int_{0}^{L} \delta(x) u_{x}^{2} d x+\frac{1}{2} \int_{0}^{L} m(x) u^{2} d x+N_{1} \tau c \int_{0}^{L}|\theta w| d x+\frac{1}{2} \int_{0}^{L} m(x) u_{t}^{2} d x \\
\leq \frac{1}{2} \int_{0}^{L} m(x) u_{t}^{2} d x+\frac{\|\delta(x)\|_{\infty}}{\lambda} \int_{0}^{L} p(x) u_{x}^{2} d x+\frac{l\|m(x)\|_{\infty}^{L}}{2 \lambda} \int_{0}^{L} p(x) u_{x}^{2} d x \\
+\frac{N_{1} \tau l c}{2} \int_{0}^{L} \theta^{2} d x+\frac{N_{1} \tau l c^{L}}{2} \int_{0}^{L} w^{2} d x \leq c^{\prime} E(t)
\end{array}
$$

where $\lambda=\operatorname{in} f_{(\mathrm{x} \in[0, L])}\{p(x)\}$, and $c^{\prime}>0$. Consequently,

$$
|\mathcal{L}(t)-N E(t)| \leq c^{\prime} E(t)
$$

which yields

$$
\left(N-c^{\prime}\right) E(t) \leq \mathcal{L}(t) \leq\left(N+c^{\prime}\right) E(t) .
$$

Choosing $N$ large enough, we obtain estimate (22).

Now, we are ready to state and prove the main result of this section.

Theorem 3.7. Let $(u, u, \theta, w)$ be the solution to system (1) - (2), then the energy E satisfies, for all $t \geq 0$,

$$
E(t) \leq c_{1} e^{-c_{2} t}
$$

where $c_{1}$ and $c_{2}$ are positive constants.

Proof. We differentiate (21), and recall (14), (16) and (18), we obtain

$$
\begin{aligned}
\mathcal{L}^{\prime}(t) \leq & N\left(-c^{\prime} \int_{0}^{L} u_{t}^{2} d x-k_{2} \int_{0}^{L} w^{2} d x-k_{3} \int_{0}^{L} w_{x}^{2} d x-k \int_{0}^{L} \theta_{x}^{2} d x\right) \\
& -\left(p\left(\xi_{1}\right)-(\eta+d) \varepsilon_{1}\right) \int_{0}^{L} u_{x}^{2} d x+m\left(\xi_{2}\right) \int_{0}^{L} u_{t}^{2} d x \\
& +\frac{\eta}{4 \varepsilon_{1}} \int_{0}^{L} \theta^{2} d x+\frac{d}{4 \varepsilon_{1}} \int_{0}^{L} w^{2} d x \\
& +N_{1}\left(\left(-k_{1} c+3 c^{\prime} \varepsilon_{2}\right) \int_{0}^{L} \theta^{2} d x+\frac{1}{2 \varepsilon_{2}} \int_{0}^{L} u_{t}^{2} d x+\frac{1}{4 \varepsilon_{2}} \int_{0}^{L} \theta_{x}^{2} d x\right) \\
& +N_{1}\left(\left(k_{1} \tau+2 \varepsilon_{2} c^{\prime}+\frac{c}{4 \dot{o}_{2}} \int_{0}^{L} w^{2} d x+\frac{1}{4 \varepsilon_{2}} \int_{0}^{L} w_{x}^{2} d x\right)\right. \\
\leq & \left.-N c^{\prime}+\frac{N_{1}}{2 \varepsilon_{2}}+m\left(\xi_{2}\right)\right\} \int_{0}^{L} u_{t}^{2} d x+\left\{-p\left(\xi_{1}\right)+(\eta+d) \varepsilon_{1}\right\} \int_{0}^{L} u_{x}^{2} d x \\
& +\left\{-N k_{2}+N_{1}\left(k_{1} \tau+2 \varepsilon_{2} c^{\prime}+\frac{c^{\prime}}{4 \varepsilon_{2}}\right)+\frac{d}{4 \varepsilon_{1}}\right\} \int_{0}^{L} w^{2} d x \\
& +\left\{N_{1}\left(-k_{1} c+3 c^{\prime} \varepsilon_{2}\right)+\frac{\eta}{4 \grave{o}_{1}} \int_{0}^{L} \theta_{0}^{2} d x+\left\{-N k+\frac{N_{1}}{4 \dot{o}_{2}}\right\} \int_{0}^{L} \theta_{x}^{2} d x\right. \\
+ & \left\{-N k_{3}+\frac{N_{1}}{4 \varepsilon_{2}}\right\} \int_{0}^{L} w_{x}^{2} d x .
\end{aligned}
$$

At this point, we choose $\epsilon_{1}$ and $\epsilon_{2}$ small enough such that

$$
-p\left(\xi_{1}\right)+(\eta+d) \epsilon_{1}<0,-k_{1} c+3 c^{\prime} \in_{2}<0,
$$

then we choose $N_{1}$ large enough so that

$$
N_{1}\left(-k_{1} c+3 c^{\prime} \varepsilon_{2}\right)+\frac{\eta}{4 \varepsilon_{1}}<0 .
$$


Once $N_{1}$ is fixed, we then choose $N$ large enough so that

$$
\begin{gathered}
-N c^{\prime}+\frac{N_{1}}{2 \grave{\dot{o}_{2}}}+m\left(\xi_{2}\right)<0 \\
-N k_{2}+N_{1}\left(k_{1} \tau+2 \varepsilon_{2} c^{\prime}+\frac{c^{\prime}}{4 \grave{O}_{2}}\right)+\frac{d}{4 \varepsilon_{1}}<0 \\
-N k+\frac{N_{1}}{4 \varepsilon_{2}}<0 \\
-N k_{3}+\frac{N_{1}}{4 \varepsilon_{2}}<0
\end{gathered}
$$

[3] Apalara TA. On the stability of porous-elastic sys-

Thus, using (13), we arrive at

$$
\mathcal{L}(t) \leq c E(t), \forall t>0
$$

A combination of (22) and (23) gives

$$
\mathcal{L}(t) \leq-c_{2} \mathcal{L}(t), \forall t>0,
$$

where $c_{2}=c / c_{2}^{\prime}$, a simple integration of $(24)$ over $(0, t)$ yields

$$
c_{1}^{\prime} E(t) \leq \mathcal{L}(t) \leq \mathcal{L}(0) e^{-c_{2} t}, \forall t>0 .
$$

Taking $c_{1}=\mathcal{L}(0) / c_{1}^{\prime}$ which completes the proof.

\section{AUTHORSHIP CONTRIBUTIONS}

Concept: M.H.; Design: M.H.; Supervision: S.Z., A.D; Data: M.H.; Analysis: M.H.; Literature Search: M.H.; Writing: M.H.; Critical Revision: A.D.

\section{DATA AVAILABILITY STATEMENT}

No new data werecreated in this study. The published publication includes all graphics collected or developed during the study.

\section{CONFLICT OF INTEREST}

The author declared no potential conflicts of interestwith respect to the research, authorship, and/or publication of this article.

\section{ETHICS}

There are no ethical issues with the publication of this manuscript.

\section{REFERENCES}

[1] Alves MS, Buriol C, Ferreira MV, Murioz Rivera JE, Sepulveda M, Vera O. Asymptotic behaviour tem with micro-temparatures, Journal of Thermal Stresses 2019;42:265-78. [CrossRef]

[4] Brezis H. Functional Analysis, Sobolev Spaces and Partial Differential Equations. Piscataway: Springer Science +Business Media, LLC; 2011. [CrossRef]

for the vibrations modeled by the standard linear solid model with a thermal effect. J Math Anal Appl 2013;399;472-9. [CrossRef]

Alves MS, Gamboa P, Gorain GC, Rambaud A, Vera O. Asymptotic behavior of a flexible structure with Cattaneo type of thermal effect. Indag Math 2016;27:821-34. [CrossRef]

[5] Casas PS, Quintanilla R. Exponential stability in thermoelasticity with micro-temperatures, International Journal of Engineering Science 2005;43:33-47. [CrossRef]

[6] Chiritea S, Ciarletta M, D'Apice C. On the theory of thermoelasticity with micro-temperatures. J Math Anal Appl 2013;397:349-361. [CrossRef]

[7] Chiritea S, Danescuca A. On the propagation waves in the theory of thermoelasticity with micro-temperatures. Mechanics Research Communications 2016;75:1-12. [CrossRef]

[8] Dridi H, Djebabla A. On the stabilization of linear porous elastic materials by microtemperature effect and porous damping, Annali Dell' Universta' Di Ferrara 2020;66:13-25. [CrossRef]

[9] Feng B, Yan L, da Silva Almeida Júnior D. Stabilization for an inhomogeneous porous-elastic system with temperature and microtemperature. Z Angew Math Mech 2020;e202000058. [CrossRef]

[10] Gorain GC. Exponential stabilization of longitudinal vibrations of an inhomogeneous beam, Nonlinear Oscil 2013;16:157-64. [CrossRef]

[11] Grot R. Thermodynamics of a continuum with microstructure. Int J Eng Sci 1969;7:801-14. [CrossRef]

[12] Hachelfi M, Djebabla A, Tatar N. On the decay of the energy for linear thermoelastic systems by thermal and micro-temperature effects. Eurasian Journal of Mathematical and Computer Applications 2018;6:29-37. [CrossRef]

[13] Iesan D. Thermoelasticity of bodies with microstructure and microtemperatures Int J Solids Struct 2007;44:8648-62. [CrossRef]

[14] Iesan D. On a theory of thermoelasticity without energy dissipation for solids with micro-temperatures. Z Angew Math Mech 2018;1-16. [CrossRef]

[15] Iesan D, Quintanilla R. On the theory of thermoelasticity with microtemperatures. J Thermal Stresses 2000;23:199-215. [CrossRef]

[16] Iesan D, Quintanilla R. On thermoelastic bodies with inner structure and micro-temperatures. J Math Anal Appl 2009;354:12-23. [CrossRef] 
[17] Iesan D, Quintanilla R. Qualitative properties in strain gradient thermoelasticity with micro-temperatures. Math Mech Solids 2018;23:240-58. [CrossRef]

[18] Li G, Luan Y, Yu J, Jiang F. Well-posedness and exponential stability of a flexible structure with second sound and time delay. Applicable Analysis 2019;98:2903-15. [CrossRef]

[19] Mageana A, Quintanilla R. Exponential stability in type III thermoelasticity with microtemperatures. Z Angew Math Phys 2018;129 69. [CrossRef]

[20] Misra S, Alves MS, Gorain G, Vera O. Stability of the vibrations of an inhomogeneous flexible structure with thermal effect Int J Dyn Control 2015;3:35462. [CrossRef]

[21] Mitrinovic DS, Pecaric JE, Fink AM. Inequalities involving functions and their integrals and derivatives. Dordrecht: Kluwer Academic Publishers, Netherlands: Springer-Science + Business Media; 1991:53.
[22] Pazy A. Semigroups of linear operators and applications to partial differential equations. Applied Mathematical Sciences 1983;44.

[23] Riha P. On the theory of heat-conducting micropolaruids with microtemperatures, Acta Mechanica 1975;23:1-8.

[24] Riha P. On the microcontinuum model of heat conduction in materials with inner structure. Int J Eng Sci 1976;14:529.

[25] Saci M, Djebabla A. On the stability of linear porous elastic materials with microtemperatures effects. Journal of Thermal Stresses 2019:1300-15. [CrossRef]

[26] A. Scalia, M. Svanadze, On the Representations of Solutions of the Theory of Thermoelasticity with Microtemperatures, Journal of Thermal Stresses 2006;29:849-63. [CrossRef]

[27] Svanadze M. Fundamental solutions of the equations of the theory of thermoelasticity with microtemperatures. Journal of Thermal Stresses 2004;27:15170. [CrossRef] 